\title{
Stochastic Cooling with a Double RF System
}

\author{
Jie Wei
}

October 1991

\section{DISCLAIMER}

\begin{abstract}
This report was prepared as an accotint of work sponsored by an agency of the United States Government. Neither the United States Guvernment nor any agency thereof, nor any of their employees, makes any warranty, express or implied, or assumes any legal liability or responsibility for the accuracy, completeness, or usefulness of any information, apparatus, product, or nricess disclosed, or represents that its use would not infringe privately owned rights. Referice herein to any specific commercial product, process, or service by trade name, trademark, minufacturer, or otherwise does not necessarily constitute or imply its endorsement, recommentation, or favoring by the United States Government or any agency thereuf. The views and opinions of authors expressed herein do not necessarily state or reflect those of the United States Government or any agency thereof.
\end{abstract}

\section{R H I C P R O J E C T}

\author{
Brockhaven National Laboratcry \\ Associated Universities, Inc. \\ Upton, NY 11973
}




\title{
STOCHASTIC COOLING WITH A DOUBLE RF SYSTEM
}

\author{
JIE WEI \\ Accelerator Development Department \\ Brookhaven National Laboratory \\ Upton, New York 11973
}

\begin{abstract}
Stochastic cooling for a bunched beam of hadrons stored in an accelerator with a double rf system of two different frequencies has been investigated. The double rf system broadens the spread in synchrotron-oscillation frequency of the particles when they mostly oscillate near the center of the rf bucket. Compared with the case of a single rf system, the cooling rate is significantly increased. When the rf voltage is raised, the reduction rate of the emittance, instead of decreasing linearly, now is independent of the ratio of the bunch area to the bucket area.

With the double rf system, the spread in synchrotron-oscillation frequency is small for particles with synchrotron-oscillation amplitudes comparable to the dimension of the rf bucket. Consequently, stochastic cooling becomes less effective when the bunch area is close to the bucket area.
\end{abstract}




\section{Introduction}

Previous studies ${ }^{1}$ indicate that stochastic cooling for a bunched beam of hadrons in a single-frequency rf system becomes difficult when the longitudinal bunch area is small compared with the area of the stable motion ( $\mathrm{rf}$ bucket area). When the bunch area is small, the spread in synchrotron-oscillation frequency is small compared with the average oscillation frequency. Compared with the coasting beam of similar line density and momentum spread, the Schottky noise in the bunched beam is higher due to the relatively higher particle density in frequency domain. Consequently, the achievable cooling rate is less. If the rf voltage is raised, the optimum cooling rate decreases linearly with the ratio of the bunch area to the bucket area (i.e. the bucket filling ratio).

Using a secondary rf system with higher frequency ${ }^{2}$ significantly broadens the spread in synchrotron-oscillation fequency of the particles near the center of the rf bucket. The achievable cooling rate can thus be significantly increased. In section II of this report, the small-amplitude particle motion in a double rf system is reviewed. Evolution of the beam under stochastic cooling is described by the Fokker-Planck equation in terms of the action variable. The reduction (cooling) rate of the transverse emittance is then obtained by an integration. In section III, the scaling behaviour of the achievable cooling rate in the double rf system is compared with that in the single rf system. Stochastic cooling in the case when the svnchrotron-oscillation amplitude is large, is discussed in section IV.

\section{Stochastic Cooling with a Double RF System}

\section{A. Equations of motion}

The voltage seen per revolution by the particle of phase deviation $\phi$ relative to the fundamental system in a double rf system, is

$$
V(\phi)=\hat{V} \sin \left(\phi+\phi_{s}\right)+k \hat{V} \sin \left(m \phi+m \phi_{2 s}\right)
$$


where

$\hat{V}=$ peak voltage of the fundamental rf system

$k \hat{V}=$ peak voltage of the higher-frequency rf system

$\phi_{s}=$ the stable phase angle relative to the fundamental rf wave-form

$\phi_{2,}=$ the stable phase angle relative to the higher frequency wave-form.

In the general case of stochastic cooling, the particle beam is stored in the accelerator without acceleràtion,

$$
\hat{V} \sin \phi_{s}+k \hat{V} \sin \left(m \phi_{2 s}\right)=0
$$

Besides, the first and second derivatives of $V(\phi)$ should vanish $^{2}$ at the center of the bunch to avoid having other stability regions nearby. Combined with Eq. 1, these conditions imply

$$
m k=1, \quad \text { and } \phi_{s}=0, m \phi_{2 s}=\pi, \quad \text { or } \phi_{s}=\pi, \phi_{2 s}=0 \text {. }
$$

Assuming $\phi_{s}=0$, and $m \phi_{2 s}=\pi$, the longitudinal motion of the particle can be described by an Hamiltonian, expressed in terms of the canonically conjugated variables $\phi$ and $W=$ $\Delta E / h \omega_{\text {a }}$ describing the deviations in rf phase and energy from the synchronous values, respectively,

$$
\mathcal{H}(\phi, W ; t)=C_{W} W^{2}+\frac{C_{\phi}}{2}(1-\cos \phi)-\frac{C_{\phi}}{2 m^{2}}(1-\cos m \phi) .
$$

Here,

$$
\begin{aligned}
& C_{W}=\frac{h^{2} \omega_{0}^{2} \eta}{2 E \beta^{2}}, C_{\phi}=\frac{q e \hat{V}}{\pi h} \\
& q e=\text { electric charge carried by the particle } \\
& h=\text { harmonic number of the fundamental rf system } \\
& \gamma_{T}=\text { transition energy } \\
& \eta=1 / \gamma_{T}^{2}-1 / \gamma^{2} \\
& \omega_{0}=\text { synchronous revolution frequency } \\
& \beta c=\text { synchronous velocity } \\
& E=m_{0} c^{2} \gamma, \text { synchronous energy. }
\end{aligned}
$$


It is known that the Hamiltonian (Eq. 4) is integrable. The so-called action variable $J$ is a constant of motion

$$
J=\oint W d \phi=\sqrt{\frac{\alpha}{C_{W}}} \oint\left\{1-\frac{C_{\phi}}{2 \alpha}\left[(1-\cos \phi)-\frac{1}{m^{2}}(1-\cos m \phi)\right]\right\}^{1 / 2} d \phi
$$

where $\alpha$ is the value of the Hamiltonian, and $0 \leq \alpha \leq C_{\phi}$. In the small-amplitude limit $m \phi \ll 1, J$ is given by

$$
J \approx \frac{8 \sqrt{2} \mathrm{~K}\left(2^{-1 / 2}\right)}{3 C_{W}^{1 / 2} C_{\phi}^{1 / 4}}\left(\frac{3}{m^{2}-1}\right)^{1 / 4} \alpha^{3 / 4}, \quad \alpha \ll C_{\phi}
$$

where $\mathrm{K}\left(2^{-1 / 2}\right) \approx 1.8541$ is the complete elliptic integral of modulus $2^{-1 / 2}$. The synchrotronoscillation frequency is given by the time derivative of the angle variable $Q$, which is canonically conjugate to $J$,

$$
\Omega_{s}=2 \pi \dot{Q}=\frac{3^{1 / 3} \pi \Omega_{0}}{2^{2 / 3} \mathrm{~K}^{4 / 3}\left(2^{-1 / 2}\right)}\left(\frac{3}{m^{2}-1}\right)^{1 / 3}\left(\frac{J}{\hat{J}_{0}}\right)^{1 / 3}, \quad J \ll \hat{J}_{0}
$$

where

$$
\hat{J}_{0}=8 \sqrt{\frac{C_{\phi}}{C_{W}}} \text { and } \Omega_{0}=\sqrt{C_{W} C_{\phi}}
$$

are the bucket area and the zero-amplitude synchrotron-oscillation frequency, respectively, in the absence of the secondary rf system. In terms of the action variable, $\phi$ and $W$ are expressed

$$
\left\{\begin{array}{l}
\phi=\hat{\phi} \operatorname{cn}\left[2 \mathrm{~K}\left(2^{-1 / 2}\right) \Omega_{s} t / \pi\right] \\
W=\hat{W} \sqrt{2} \operatorname{sn}\left[2 \mathrm{~K}\left(2^{-1 / 2}\right) \Omega_{s} t / \pi\right] \operatorname{dn}\left[2 \mathrm{~K}\left(2^{-1 / 2}\right) \Omega_{s} t / \pi\right]
\end{array}\right.
$$

where sn, cn, and dn are the Jacobian elliptic functions., ${ }^{3,4}$ Here in Eq. 9, the amplitudes of the oscillation are

$$
\hat{\phi}=2\left(\frac{3}{m^{2}-1}\right)^{1 / 4}\left(\frac{\alpha}{C_{\phi}}\right)^{1 / 4}, \quad \hat{W}=2\left(\frac{\alpha}{C_{W}}\right)^{1 / 2}
$$

where $\alpha$ is related to $J$ by Eq. 6 .

Eq. 7 indicates that a secondary rf system changes the spread in synchrotron-oscillation frequency of the particles of different oscillation amplitudes. Because of this change, the 
structure of the synchrotron side-bands in the frequency domain becomes different. For a typical bunch of particles where the density is the highest at the center, and zero at the separatrix, the side-band splitting no longer exists. On the other hand, side-band overlapping becomes significant.

\section{B. The transport equation for stochastic cooling}

Consider the stochastic cooling of the transverse dimension $x$ of a bunched beam of $\underset{x}{N}$ particles that perform synchrotron oscillation with frequencies $\Omega_{i}$ and phase amplitudes $\hat{\phi}_{i}$. The increment $U_{x^{\prime}}$ in $x^{\prime}=d x / d s$, which is experienced by the particle $i$ per unit time at the kicker, is proportional to the displacement $x^{P}$ of all the particles at the pick-up, ${ }^{1}$

$$
U_{x^{\prime}, i}=\sum_{j=1}^{N} U_{i j} x_{j}^{P}
$$

where

$$
\begin{aligned}
U_{i j}= & \frac{f_{0}^{2}}{\sqrt{\beta_{x}^{P} \beta_{x}^{K K}}} \sum_{m=-\infty}^{\infty} \sum_{l=-\infty}^{\infty} i^{l} J_{l}\left(-m \hat{\phi}_{j} / h\right) \exp \left(i l \phi_{j}^{0}\right)\left(x\left(m^{ \pm} \omega_{0}-l \Omega_{j}\right)\right. \\
& \times \sum_{n=-\infty}^{\infty} \sum_{k=-\infty}^{\infty} i^{k} J_{k}\left(-n \hat{\phi}_{i} / h\right) \exp \left[i t\left(m \omega_{0}-l \Omega_{j}+n \omega_{0}-k \Omega_{i}\right)+i k \phi_{i}^{0}\right]
\end{aligned}
$$

Here, $G(\omega)$ is the gain of the cooling system, $\beta_{x}$ is the Courant-Snyder parameter, $J_{l}$ is the Bessel function of $l$ th order, and $\phi^{0}$ is the initial phase of synchrotron oscillation. The superscript $P$ and $K$ denote values at the pick-up and kicker, respectively.

It is convenient to describe the transverse motion of the particles in terms of the transverse angle-action variables $\varphi$ and $I$, which are generated from the original variables $x$ and $x^{\prime}$ by a generating function of Goldstein's first type ${ }^{5}$

$$
F_{1}(x, \varphi ; s)=-\frac{x^{2}}{2 \beta_{x}}\left(\tan \varphi-\frac{\beta_{x}^{\prime}}{2}\right)
$$

The equations of motion then become

$$
\left\{\begin{array}{l}
\dot{\varphi}=\frac{1}{\beta_{x}}+U_{\varphi} \\
\dot{I}=U_{I}
\end{array}\right.
$$


where

$$
U_{I}=-\sqrt{2 \beta_{x} I} \sin \varphi U_{x^{\prime}}, \quad U_{\varphi}=-\sqrt{\beta_{x} / 2 I} \sin \varphi U_{x^{\prime}} .
$$

Typically, the time for stochastic cooling to produce an appreciable effect is much longer than the revolution period. The evolution of the transverse distribution function $\Psi$ of the particles can be described by the transport equation, which is obtained by averaging the two-dimensional Fokker-Planck equation over $\varphi$

$$
\frac{\partial \Psi}{\partial t}=-\frac{\partial}{\partial I}(F \Psi)+\frac{1}{2} \frac{\partial}{\partial I}\left(D \frac{\partial \Psi}{\partial I}\right) .
$$

Neglecting the thermal noise of the cooling system, the coefficients of coherent correction $F$ and diffusion $D$ can be evaluated by employing the representation of the Jacobian elliptic function ${ }^{4}$ as a trigonometric series

$$
\operatorname{cn}\left[2 \mathrm{~K}\left(2^{-1 / 2}\right)\right]=\cos \Omega_{s} t\left[1-4 \xi \sin ^{2} \Omega_{s} t+O\left(\xi^{2}\right)\right], \quad \xi=e^{-\pi}
$$

and keeping only the leading term. Then,

$$
F(I)=F^{0} I, \quad D_{S H}=D^{0} I\langle I\rangle .
$$

Here,

$$
\begin{aligned}
F^{0}= & -f_{0}^{2} \int d J \rho(J) \sin \nu_{x} \Delta \theta^{P K} \sum_{m=-\infty}^{\infty} \sum_{l=-\infty}^{\infty} G\left(m^{ \pm} \omega_{0}-l \Omega_{i}\right) e^{i l \Omega_{i} \Delta \theta^{P K} / \omega_{0}} J_{l}^{2}\left(m \omega_{0} \tau_{i}\right) \\
D^{0}= & \left.\pi f_{0}^{4} \int d J \rho(J) \sum_{l=-\infty}^{\infty} \sum_{k=-\infty}^{\infty} \frac{\rho\left(J^{\prime}\right)}{|l|\left|\frac{d \Omega_{s}\left(J^{\prime}\right)}{d J^{\prime}}\right|}\right|_{\Omega_{\odot}\left(J^{\prime}\right)=k \Omega_{\odot}(J) / l} \\
& \times\left\{\left|G_{D}(-)\right|^{2}+\left|G_{D}(+)\right|^{2}+2 \operatorname{Re}\left[G_{D}(-) G_{D}(+)\right]\right\}
\end{aligned}
$$

with

$$
G_{D}( \pm)=\sum_{m=1}^{\infty} G\left(m^{ \pm} \omega_{0} \pm l \Omega_{j}\right) J_{\mp l}\left(m \hat{\phi}_{j} / h\right) J_{\mp k}\left(m \hat{\phi}_{i} / h\right)
$$

The boundary condition to this equation is

$$
\left\{\begin{array}{l}
I=0: \quad-F \Psi+\frac{D}{2} \frac{\partial \Psi}{\partial I}=0 \\
I=I_{\text {apt }}: \quad \Psi=0
\end{array}\right.
$$


where $I_{\text {apt }}$ is the transverse aperture of the accelerator. In Eq. 19, $\rho(J)$ is the density in longitudinal action $J$, and the rate of change of synchrotron-oscillation frequency with respect to $J$

$$
\frac{d \Omega_{s}(J)}{d J}=\frac{3^{1 / 3} \pi C_{W}}{242^{2 / 3} \mathrm{~K}^{4 / 3}\left(2^{-1 / 2}\right)}\left(\frac{m^{2}-1}{3}\right)^{1 / 3}\left(\frac{\hat{J}}{J}\right)^{2 / 3} .
$$

approaches infinity near the center of the bucket $(J=0)$.

\section{Optimum cooling rate}

Because $F$ and $D$ are both independent of $\Psi$, the reduction rate of the transverse emittance can be obtained by integrating Eq. 16,

$$
\tau^{-1}=\frac{1}{\langle I\rangle} \frac{d\langle I\rangle}{d t}=F^{0}+\frac{D^{0}}{2} .
$$

From Eq. 23, the average gain $G_{\text {opt }}$ for achieving the optimum cooling rate can be obtained. Because the number of significant synchrotron sideband $\langle k\rangle=\langle n\rangle \omega_{0}\langle\tau\rangle$ is typically much larger than 1, $J_{\mp k}$ in Eq. 20 can be replaced by its asymptotic form. Employing the identity ${ }^{3}$

$$
J_{0}^{2}(x)+2 \sum_{k=1}^{\infty} J_{k}^{2}(x)=1
$$

the optimum gain can be derived

$$
G_{o p t}^{-1}=\frac{\Delta n f_{0}\left\langle\rho\left(\Omega_{j}\right)\right\rangle}{\pi\langle k\rangle\langle n\rangle\langle\tau\rangle}
$$

Here, \langle\rangle denotes the average over the quantity $J,\langle n\rangle$ is the average harmonic of the cooling system, and $\Delta n f_{0}$ is the frequency bandwidth. By employing Eqs. 22 and 9 , the optimum rate can be approximately obtained

$$
\tau_{o p t}^{-1}=\frac{\langle k\rangle^{2}}{2\langle\rho(J)\rangle}\left\langle\left|\frac{d \Omega_{s}}{d J}\right|\right\rangle \approx \frac{\pi\langle n\rangle^{2} C_{W}}{8 \mathrm{~K}^{2}\left(2^{-1 / 2}\right) h^{2}\langle\rho(J)\rangle}
$$

which is valid for small-amplitude oscillations. Note that in obtaining Eq. 26, synchrotron side-band overlapping has been neglected.

A computer program has been developed to evaluate the cooling rate with the double rf system. The coefficients $\left(F^{0}\right.$ and $\left.D^{0}\right)$ of the transport equation are eviluated according 


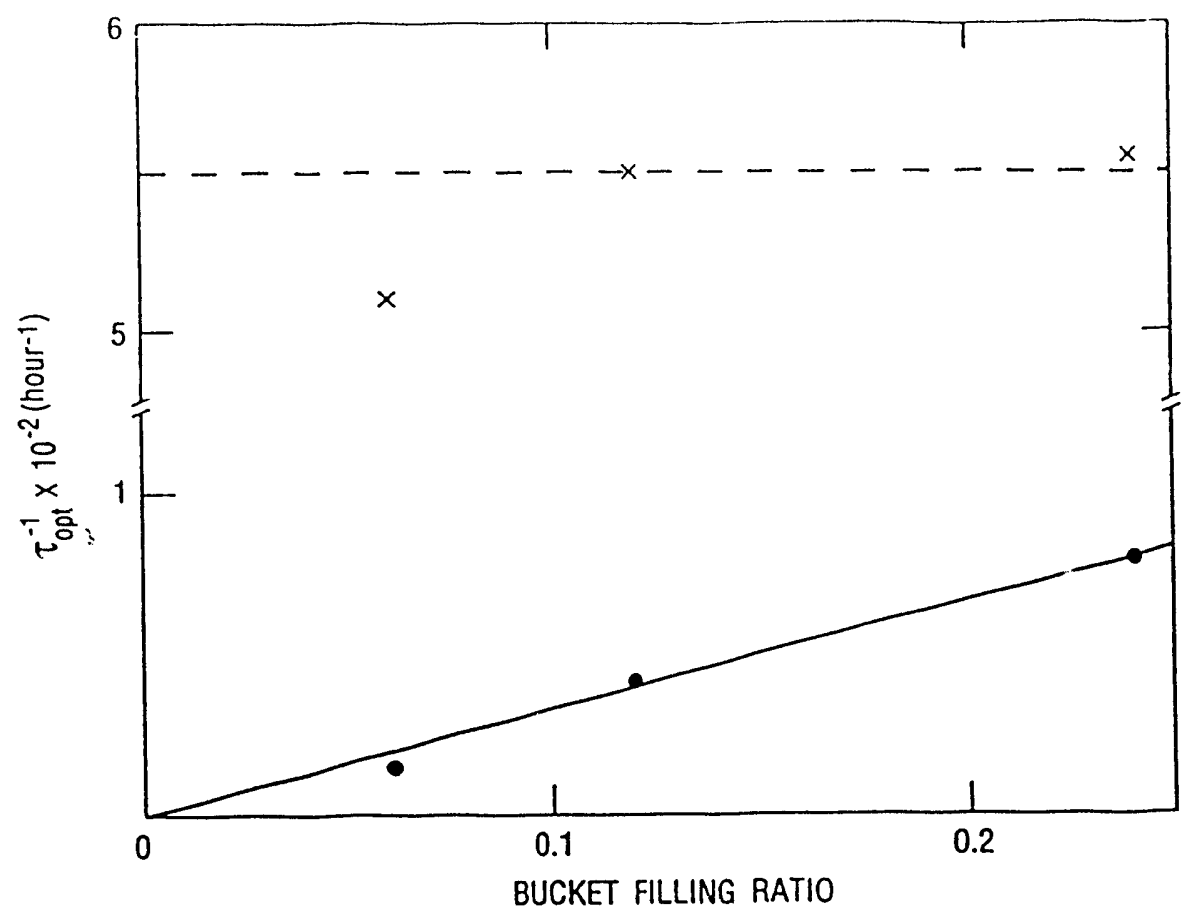

Figure 1: The reduction rates of the transverse emittance as a function of the bucket filling ratio for a bunch of ${ }^{197} \mathrm{Au}^{79+}$ ions stored with a single (solid line) and a double (dashed line) rf system, respectively.

to Eq. 19 including synchrotron side-band overlapping. Fig. 1 shows the transverse cooling rates of a bunch of $10^{9}{ }^{197} \mathrm{Au}^{79+}$ ions during stochastic cooling in the RHIC. ${ }^{6}$ The bunch is assumed to be a truncated Gaussian with a $95 \%$ area of $0.3 \mathrm{eV} \cdot \mathrm{s} / \mathrm{u}$. The frequency bandwidth of the cooling system is 4,-8 GHz. The dashed line indicates that with a fundamental system of $160 \mathrm{MJHz}$ and a secondary system of twice the frequency $(m=2)$, the cooling rate is independent of the bucket filling ratio.

\section{Comparison with Single RF System Cooling}

Stochastic cooling with a single rf system has been investigated previously. ${ }^{1}$ The optimum rate of reduction of the transverse emittance is

$$
\tau_{\text {opt }}^{-1} \approx \frac{\langle n\rangle^{2} C_{W}}{\pi^{2} h^{2}\langle\rho(J)\rangle} \frac{\langle J\rangle}{\hat{J}_{0}}, \quad J<\hat{J}_{0} .
$$

In the case that the particle distribution in $J$ remains unchanged, the transverse cooling 
rate is proportional to the bucket filling ratio. For a certain bunch area, cooling becomes less effective when the rf voltage is raised. The solid line in Fig. 1 shows the same transverse cooling rate as the dashed line, except with a single rf system of $160 \mathrm{MHz}$. The cooling rate decreases linearly with the increasing bucket area.

The dashed line in Fig. 1 shows that the cooling efficiency can be significantly improved with a secondary if system, especially for cases of small bucket filling ratio. When the secondary rf system is employed, the spread in synchrotron-oscillation frequency is broadened appreciably. Eq. 26 indicates that the optimum cooling rate is approximately independent of the variation of the rf voltage if the synchrotron-oscillation amplitude is small.

\section{Cooling in the Full Bucket Case}

In the case of a single rf system, the spread in synchrotron-oscillation frequency increases for particles of large synchrotron-oscillation amplitudes. Consequently, stochastic cooling becomes more effective when the particles occupy larger amount of the rf bucket.

On the contrary, cooling is less effective in the case of a double rf system when the if bucket becomes full. Consider the example that the frequency of the secondary system is twice that of the fundamental system $(m=2)$. The synchrotron-oscillation frequency can be derived

$$
\Omega_{s}=\frac{\pi \Omega_{0}}{\sqrt{2} \mathrm{~K}(\xi)}\left(\frac{\alpha}{C_{\phi}}\right)^{1 / 4}, \quad \xi=\sqrt{\frac{1}{2}\left(1+\sqrt{\frac{\alpha}{C_{\phi}}}\right)} .
$$

As shown in Fig. 2, $\Omega$, vanishes both at the bucket center and at the separatrix. The rate of change of the frequency with respect to $J$ is given by

$$
\frac{d \Omega_{s}(J)}{d J}=\frac{\pi \Omega_{0}}{2 \hat{J}_{0} \mathrm{~K}^{2}(\xi)}\left(\frac{\alpha}{C_{\phi}}\right)^{1 / 2}\left[\frac{C_{\phi}}{\alpha}+\frac{1}{\sqrt{\frac{\alpha}{C_{\phi}}}\left(1+\sqrt{\frac{\alpha}{C_{\phi}}}\right)}-\frac{2}{\sqrt{\frac{\alpha}{C_{\phi}}}\left(1-\frac{\alpha}{C_{\phi}}\right)} \times \frac{\mathrm{E}(\xi)}{\mathrm{K}(\xi)}\right]
$$

as shown in Fig. 3. In these equations, the action $J$ is related to $\alpha$ by

$$
J=\int_{0}^{\alpha} \frac{2 \pi}{\Omega_{s}\left(\alpha^{\prime}\right)} d \alpha^{\prime}
$$

as shown in Fig. 4. It is indicated from Figs. 2 and 3 that for particles with large 


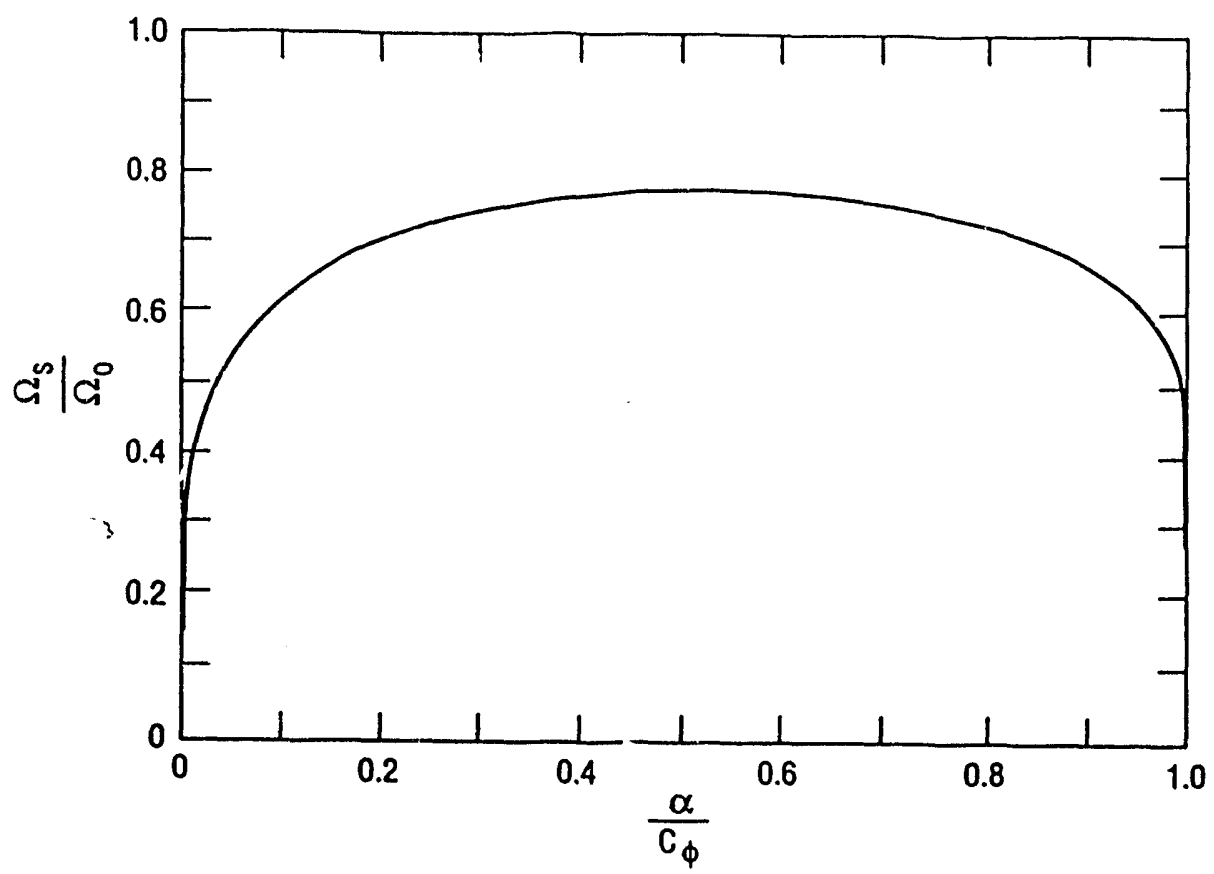

Figure 2: The synchrotron-oscillation frequency as a function of the normalized Hamiltonian in the $(m=2)$ double rf system.

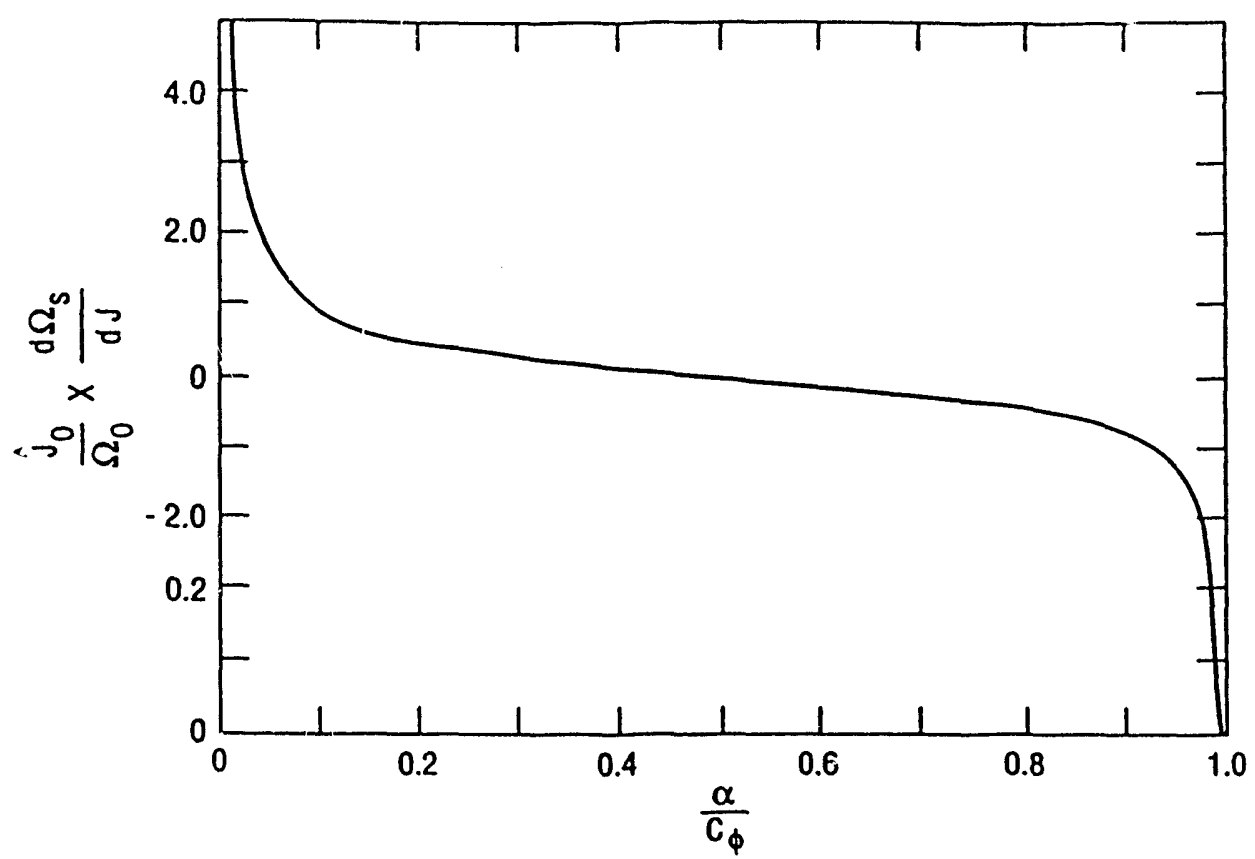

Figure 3: The quantity $d \Omega_{\mathbb{s}} / d J$ as a function of the normalized Hamiltonian in the $(m=2)$ double rf system. 


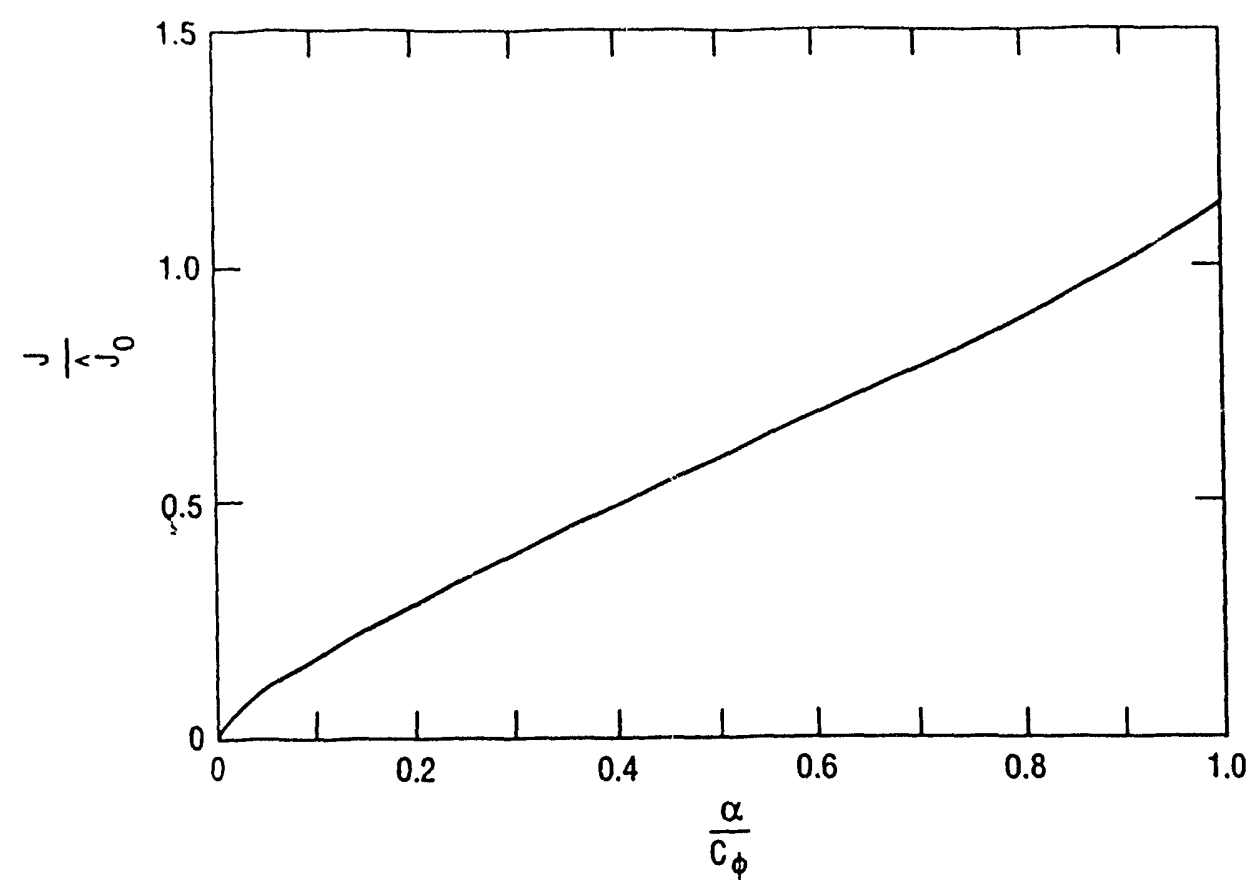

Figure 4: The action $J$ as a function of the normalized Hamiltonian in the $(m=2)$ double rf system.

synchrotron-oscillation amplitude (approximately with $J / \hat{J}_{0}$ between 0.3 and 0.8 ), the spread in synchrotron-oscillation frequency is small. Stochastic cooling becomes difficult if most of the particles in the bunch are in this large-amplitude region.

\section{Conclusion}

Stochastic cooling for a bunched beam of hadrons stored with a double rf system of two different frequencies has been investigated. The double if system broadens the spread in synchrotron-oscillation frequency of the particles when they are near the bucket center. This broadening in frequency effectively decreases the Schottky noise. Compared with the case of a single rf system, the achievable cooling rate is significantly increased. When the If voltage is raised, the reduction rate of the emittance, instead of decreasing linearly, now is independent of the bucket filling ratio.

On the other hand, the spread in synchrotron-oscillation frequency becomes small if the 
oscillation amplitudes of the particles arc comparable to the bucket dimension. Stochastic cooling with the double rf system is less effective when the bunch area is close to the bucket area.

\section{Acknowledgments}

The authos would like to thank Drs. J. Marriner and G. Jackson for suggesting the problem, Drs. S. Ohnuma and A. Hofmann for discussion, and Dr. H. Hahn for reading the manuscript.

\section{REFERENCES}

1. J. Wei, Comparison between coasting and bunched beams on optimum stochastic cooling and signal suppression, Proc. 1991 Particle Accelerator Conference, San Francisco (1991, to be published). (BNL-45515)

2. A. Hofmann and S. Myers, Beam dynamics in a double rf system, Proc. 11th Int. Conf. on High Energy Accelerators, CERN (1980) 610.

3. I.S. Gradshteyn and I.M. Ryshik, Table of Integrals, Series, and Products (Academic, New York, 1965).

4. E. Jahnke and F. Emde, Tables of Functions with formulae and curves (Dover, New York, 1945), p92.

5. H. Goldstein, Classical Mechanics (Addison-Wesley, New York, 1953).

6. Brookhaven National Laboratory, Conceptual Design of the RHIC, BNL-52195 (1989). 

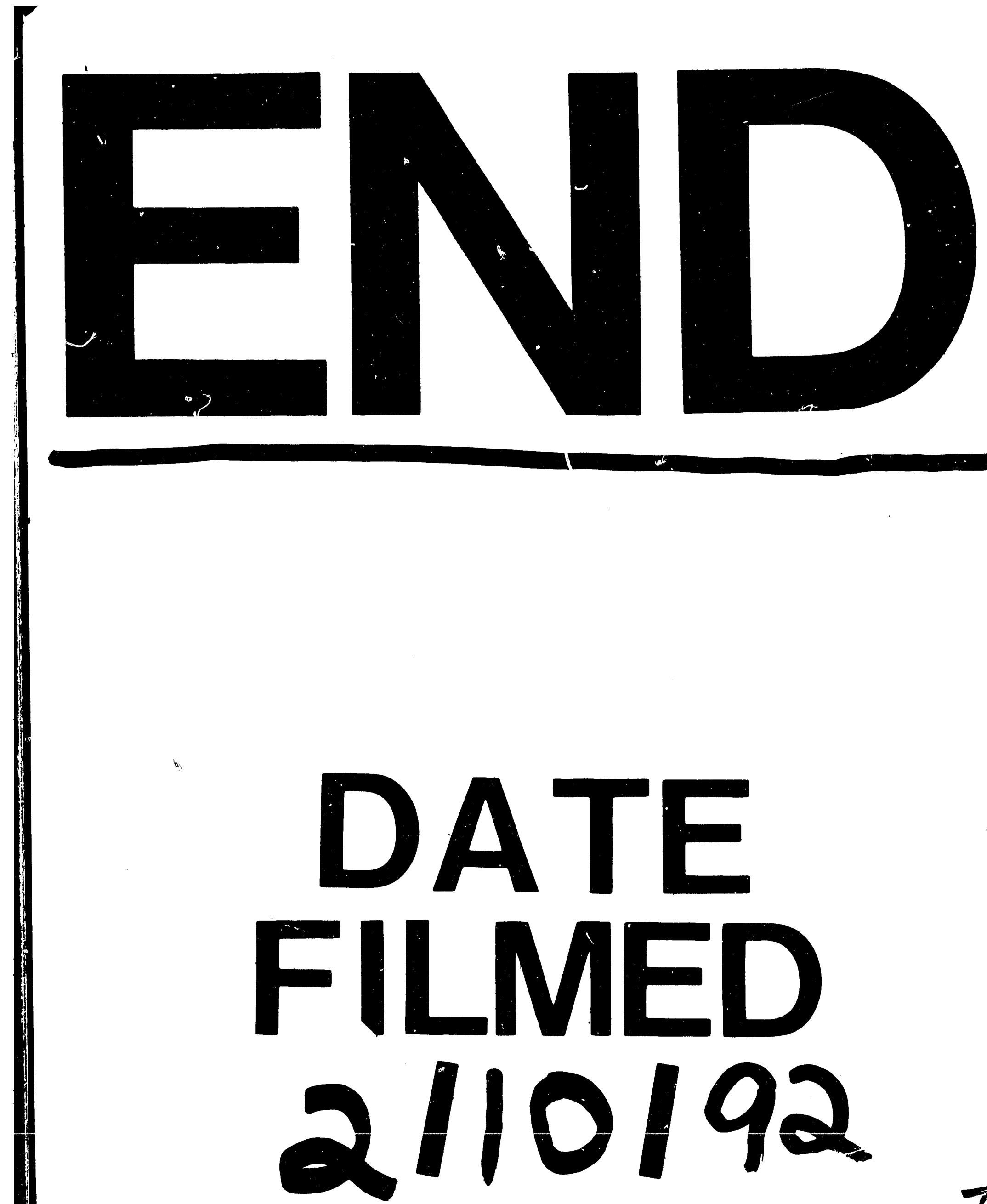

$I$ 
